\title{
The Prevalence of Post-Traumatic Stress Disorder in Self- Identified South Asians
}

\author{
Sruthi K. Medepalli \\ Lynbrook High School,1280 Johnson Ave, San Jose, CA, 95129, USA; sruthi.medepalli@gmail.com
}

ABSTRACT: Post-traumatic stress disorder (PTSD) is widely regarded as a post-war veteran's disease, a stereotype that this study aims to dispel. The goal of this study was to determine the prevalence of this disorder in understudied populations, specifically in the South Asian population. Firstly, the prevalence of post-traumatic stress disorder (PTSD) in various geographical areas was determined using existing datasets. This experimental approach to determining PTSD prevalence used the PCL-C self-assessment and scoring based on the Diagnostic and Statistical Manual of Mental Disorders (DSM-5) criterion. 40 adult subjects of South Asian descent living in the U.S., Canada, and India were randomly selected and their anonymous responses to the PCL-C questions were analyzed. The results showed that $37.5 \%$ of the subjects had moderate to very severe levels of PTSD. This suggests that there may be a higher percentage of PTSD in South Asians than previously recognized. This study was limited by the fact that the South Asians within this study were self-identified and that the study had a small sample. However, due to the inadequate amount of existing research on anxiety and trauma/stressor-related disorders in this subject population, it is highly recommended that a broader and deeper study of PTSD prevalence, causes, and treatment in this population be examined.

KEYWORDS: Behavioral and Social Sciences; Neuroscience; Mental Disorders; Post-Traumatic Stress Disorder; South Asia.

\section{Introduction}

One in eleven people will be diagnosed with PTSD at some point in their lives, and it affects about 8 million people per any given year. ${ }^{1}$ Soldiers and veterans are most susceptible to trauma, and therefore, to PTSD. But this "soldier's disease" has been given multiple names in its past, including "shell-shock" during World War I and "combat fatigue" in the time of World War II. Although these descriptions corresponded to the same disease, the idea that PTSD only affected war veterans was erroneous.

\section{Causes and Risk Factors:}

Risk factors for PTSD include histories of substance abuse, mental illness, physical and sexual assault, high-stress level in daily life, lack of a support system, and poor coping mechanisms. Women are twice as likely to get PTSD simply for the reason that they are a more common target for sexual assault and rape. ${ }^{2}$

Family history is one of the biggest factors for mental illnesses and neurodegenerative disorders, and PTSD is no different. Susceptibility to PTSD also has genetic components and environmental effects as a result of trauma, that

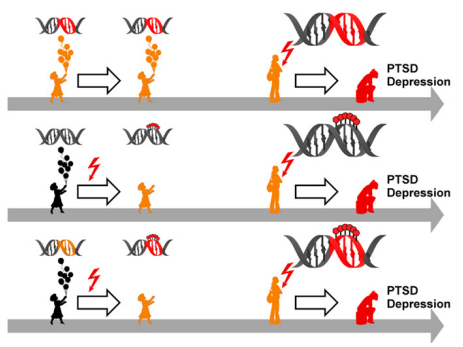

Figure 1: Diagram of Genetic PTSD. ${ }^{3}$ leaves a chemical (epigenetic) mark on your genes, as illustrated in Figure 1.

\section{Diagnosis and Brain Function:}

When diagnosing patients for PTSD, clinicians often look for detachment, reexperiencing the traumatic event, emotional effects, avoidance, and sympathetic hyperactivity (hypervigilance), most of which must span a month or longer. More specifically, physicians often examine the amygdala, prefrontal cortex, and hippocampus. Indicators of PTSD come from immune, genetic, hormonal, and diagnostic factors. The amygdala is used for fear, and PTSD affected brains often over-panic and have heightened fear reactions. The prefrontal cortex processes and regulates the emotional reactions coming from the amygdala, and it often does not function well when a patient has PTSD because of their tendency to be over/under emotional. Finally, the hippocampus is the memory center of the brain, and the PTSD-affected brain has difficulty remembering the event and often constantly replays specific memories of the trauma. ${ }^{4}$

As with other psychological disorders, physicians often refer to the DSM-5. The manual, as written by the American Psychological Association, lists seven criteria that must be met in response to the first criteria, a stressor. ${ }^{5}$ Criterion A is arguably one of the most essential to be met, as most other criteria overlap with those of general anxiety disorders and phobias, as well as acute stress disorder. A stressor is defined as an event in which one has been exposed to death, threatened death, actual or threatened sexual violence. Criterions B and $\mathrm{C}$ include intrusion symptoms and avoidance, with at least one of each required. These are defined as the traumatic event being persistently reexperienced in memories, nightmares, flashbacks, emotional distress, physical reactivity to trauma 
reminders respectively. Criterion $\mathrm{D}$, or negative alterations in cognitions and mood, is negative thoughts/feelings that have worsened after trauma in inability to recall key features of the trauma, exaggerated blame, decreased interest, and isolation. Two of these are required for a patient to be properly diagnosed. Criterion $\mathrm{E}$ is closely related, being alternations in arousal and activity. This is shown through worsened behavior such as irritability, risky or destructive behavior, heightened startle reaction, difficulty sleeping and concentrating. Criteria $\mathrm{F}, \mathrm{G}$, and $\mathrm{H}$ are duration, exclusion, and distress. These require that symptoms last for more than a month, are not due to substance abuse, medication, or mental illness, and cause distress or functional impairment.

\section{Screening and Treatment:}

Current brain-screening techniques include Positron Emission Topography (PET) scans of mGluR5 receptor availability, which is a type of brain cell receptor for the neurotransmitter glutamate, that is altered in PTSD patients. Figure 2 showcases the PET scan of a PTSD affected brain.

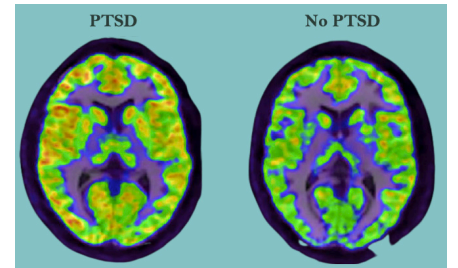

Figure 2: PET Scan of PTSD Afflicted Brain vs. Healthy Brain. ${ }^{6}$

Magnetic Resonance Imaging (MRI) scans assess the effects of stroke, trauma, and degenerative diseases and can be used on PTSD patients to reveal grey-matter reductions in certain parts of the brain, as demonstrated in Figure 3 (see scan on the left).

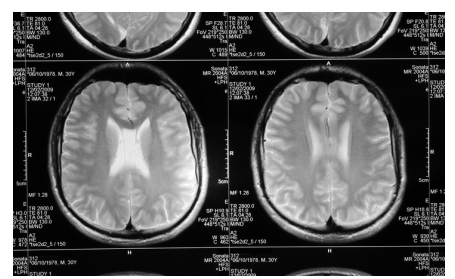

Figure 3: MRI Scan of Healthy Brain vs. PTSD Affected Brain. ${ }^{7}$

The current most productive treatment is therapy-based, specifically Cognitive-Behavioral Therapy (CBT) and Eye Movement Desensitization \& Reprocessing (EMDR). Other common forms of psychotherapy include exposure therapy, cognitive restructuring therapy, and group therapy. Therapists employing CBT encourage patients to re-evaluate their thinking patterns and assumptions to identify unhelpful patterns in thought, like overgeneralizing bad outcomes, negative thinking, and expectations of catastrophic outcomes, into more positive and effective thinking patterns. CBT is meant to help the person rethink their understanding of traumatic experiences, themselves, and their ability to cope. The goal of exposure therapies for the patient will actively confront fears and emotions, learning along the way that anxiety and fear will lessen on their own. The goal is to eliminate avoidance and increasing quality of life. EMDR therapy accesses the traumatic memory network in order to enhance information processing, forming new associations between the traumatic memory and more adaptive memories or information. Trau$\mathrm{ma}$ is often stuck in the limbic system of the brain, consisting mainly of the hippocampus, amygdala, hypothalamus, and thalamus, and which is known as the emotional brain. EMDR focuses specifically on these areas. These result in elimination of emotional distress and development of cognitive insight.

Pharmacological treatments can also be effective, and usually aim to reduce specific symptoms. Symptoms of PTSD overlap with those of depression and anxiety; therefore, antidepressants and anti-anxiety medication are feasible for patients. Anti-depressants focus on the production of three chemicals in the brain- serotonin, norepinephrine, and oxytocin. Ongoing and completed clinical trials have investigated the effect that music therapy, ${ }^{8}$ cannabis, ${ }^{9}$ and medications including hydrocortisone, morphine, oxytocin, propranolol, and benzodiazepines. ${ }^{10}$ Understanding the differences in approaching trauma and targeting symptoms is vital to a full understanding of the disorder.

\section{Geography:}

Current research in PTSD based on geographic location shows a very limited reach, and studies generally only research into countries in Europe and the Americas. Countries like Canada show the highest PTSD-affected population, at $9.20 \%,{ }^{11}$ while China has the lowest rate, with only $0.30 \%{ }^{12}$ reported with PTSD. A geographical compilation of existing PTSD research is encapsulated in Figure 4. As seen there, the South Asian region has been greyed out, only limited studies have been done in the villages surrounding Pakistan, Bangladesh, and India investigating the effects of low natural resources and disasters on PTSD. ${ }^{13}$ In contrast, this research focuses on the prevalence of PTSD in the South Asian ethnic pool.

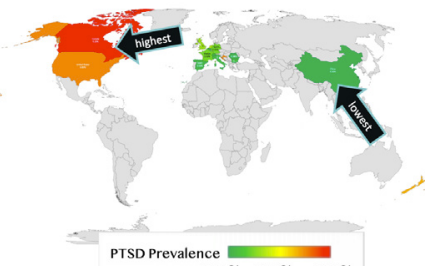

Figure 4: PTSD Prevalence.

\section{Methods}

The PCL-C self-assessment was used based on the DSM-5 PTSD symptom requirements. The exact measures and questions from the official assessment were entered onto an online form, allowing participants to be completely anonymous. Therefore, there was no requirement to submit the methods to a research review board. This study assumed the presence of a stressor, or Criterion A (Diagnosis and Brain Function) in each participant, given the variance in the answers received when asking for participants to recall a traumatic event. Hence, the eventual prognoses may lack certainty in associating stressor type and severity with a specific individual. The anonymous surveys were conducted from August 2020 to September 2020 by sending the online form to social media groups dominated by South Asians and a random pool of subjects voluntarily responded to it. The subjects 
spread across the US, Canada and India (12 respondents were from US, 18 from Canada, 10 were from India). The subject age group were adults 18 and older. Due to the sensitive nature of this survey, the privacy of the subjects has been strictly enforced, hence no attempt has been made to correlate subjects with underlying health problems or genetic history. However, none of the subjects had prior diagnosis or awareness of PTSD. The 40 subjects were assigned patient numbers and each one was diagnosed. This was done by adding together their given responses from a range of 1-4 to 20 questions. From there, the percentage of subjects with mild, moderate, severe, and very severe PTSD were found. ${ }^{14}$ The control group was composed of subjects with scores from 2129. Those who scored between 30-35 were diagnosed with mild PTSD, 36-45 was moderate, $45-55$ was severe, and subjects who scored above 56 had very severe PTSD.

\section{- Results and Discussion}

Table 1 summarizes the survey results grouped by PTSD severity. The most common group within our subjects was the control group, or individuals with no official PTSD diagnosis accounting for $37.5 \% .15 \%$ were diagnosed with mild PTSD, $17.5 \%$ with moderate, $7.5 \%$ with severe PTSD, and an alarming $12.5 \%$ with very severe PTSD. This means that the percentage of subjects with severe and very severe PTSD was in total, 20\%. Figure 5 graphs the severity of the subjects overall in order to gain a fuller understanding of the distribution of scores.

Table 1: PTSD Scores Received.
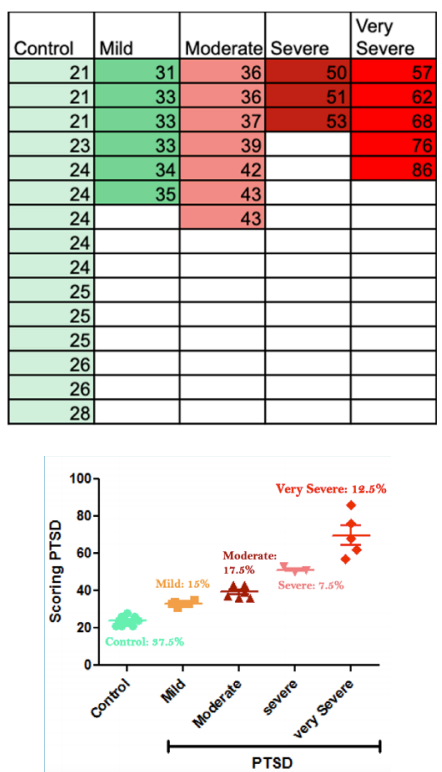

Figure 5: PTSD Scores Organized by Severity.

This experimental study also showed that although $3.5 \%$ of U.S. citizens experience PTSD, the average score of subjects in the U.S. was 38, indicating moderate to severe PTSD. Furthermore, past research shows that $9.20 \%$ of Canadian citizens have been diagnosed with PTSD, and in this study, it was observed that Canada had sufficiently lower scores than the average of the U.S. Figure 6 shows the geographical prevalence of PTSD in the subjects. Based on the data collected from every subject which self-identified as being of South Asian, descent, the scores received were all sufficiently higher than the 5.2 percent average worldwide. ${ }^{15}$

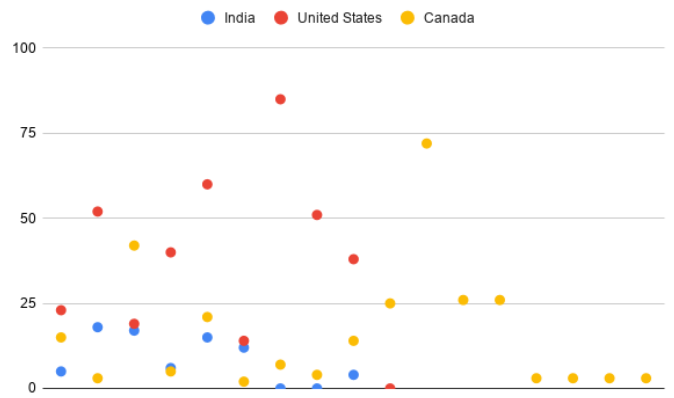

Figure 6: PTSD Scores Organized by Geographys.

These scores were potentially affected by the stress of the coronavirus pandemic, which gave families worldwide trauma from businesses closing, death of loved ones, and even having the virus themselves.

\section{Conclusion}

The prevalence of PTSD in the South Asian population and in the coronavirus-affected world is disproportionately large, indicating further studies into these particular subject groups. Having a realistic idea of how common trauma and mental disorders is necessary for the general public and for patients. With stress about working conditions, personal/ family health, and online school, the entire population has become more susceptible to trauma. $20 \%$ of the subjects in this study were diagnosed with severe to very severe PTSD with no previous diagnosis or knowledge of PTSD, showing how often and undiscussed this disorder is. This $20 \%$ average, although within a relatively small sample size, shows a clear advance from the 5.2\% of PTSD-affected people worldwide. Previous research focuses mainly on areas within Europe and North America, disregarding Asian countries, despite the level of PTSD there and the universal nature of PTSD exposure - PTSD can affect anyone. Hence, it is strongly recommended that future studies are conducted with a focus in this ethnic group. Larger sample sizes within these areas are highly encouraged in order to gain a better understanding. Finally, understanding the effect this pandemic has had on humans' mental state and risk towards trauma is also essential to helping everyone recover from this past year - for example, repeating this survey with the same subjects after the COVID pandemic has ended globally.

\section{- Acknowledgements}

I am incredibly grateful to my mentor, Dr. Shrivastava, for helping me through this process, to SkoolMentor for allowing me access to this opportunity, and to my entire family, for continuing to support me in my endeavors. I would also love to thank the reviewers, whose advice and comments contributed so much to the contents of this paper and to my own understanding of psychiatric disorders.

\section{References}

1. "What Is Posttraumatic Stress Disorder?"What Is PTSD?, American Psychiatric Organization, www.psychiatry.org/patientsfamilies/ptsd/what-is-ptsd. "VA.gov: Veterans Affairs." 
2. How Common Is PTSD in Women?, U.S. Department of Veteran's Affairs, 18 Sept. 2018, www.ptsd.va.gov/understand/common/ common_women.asp\#: :text=After $\% 20 \mathrm{a} \% 20$ trauma $\% 2 \mathrm{C} \% 20$ some\%20women,likely\%20to\%20 experience\%20sexual\%20assault.

3. Raabe, Florian \& Spengler, Dietmar. (2013). Epigenetic Risk Factors in PTSD and Depression. Frontiers in psychiatry. 4. 80. 10.3389/fpsyt.2013.00080.

4. Bremner JD. Traumatic stress: effects on the brain. Dialogues Clin Neurosci. 2006;8(4):445-461. doi:10.31887/DCNS.2006.8.4/ jbremner.

5. Center for Substance Abuse Treatment (US). Trauma-Informed Care in Behavioral Health Services. Rockville (MD): Substance Abuse and Mental Health Services Administration (US); 2014. (Treatment Improvement Protocol (TIP) Series, No. 57.) Exhibit 1.3-4, DSM-5 Diagnostic Criteria for PTSD. Available from: https://www.ncbi.nlm.nih.gov/books/NBK207191/box/part1_ ch3.box16/

6. Sophie E. Holmesa,b, Matthew J. Girgentia,b,c, Margaret T.Davisa,b, Robert H. Pietrzaka,b,c, Nicole DellaGioiaa,b, Nabeel Nabulsia,b, David Matuskeya,b, Steven Southwicka,b,c, Ronald S. Dumana,b,c, Richard E. Carsona,b, John H. Krystalaa,b,c, Irina Esterlisa,b,c, and the Traumatic Stress Brain Study Group, 2017 Altered metabotropic glutamate receptor 5 markers in PTSD: in vivo and postmortem evidence. Proceedings of the National Academy of Sciences (USA) 114: 8390-8395.

aDepartment of Psychiatry, Yale University School of Medicine, New Haven, CT 06519;

bDepartment of Radiology and Biomedical

Imaging, Yale University School of Medicine, New Haven, CT 06519; and cNational Center for PTSD, Clinical Neurosciences Division, West Haven, CT 06515

7. Stoppelbein, L., Greening, L., \& Fite, P. (2017). The role of cortisol in PTSD among women exposed to a trauma-related stressor. Journal of Anxiety Disorders, 26(2), 352-358. https://doi. org/10.1016/j.janxdis.2011.12.004

8. "Guitars for Vets: Evaluating Psychological Outcome of a Novel Music Therapy”, Sponsored by US Department of Veterans Affairs, 2015 April 7

9. Orsolini L, Chiappini S, Volpe U, Berardis D, Latini R, Papanti GD, Corkery AJM. Use of Medicinal Cannabis and Synthetic Cannabinoids in Post-Traumatic Stress Disorder (PTSD): A Systematic Review. Medicina (Kaunas). 2019 Aug 23;55(9):525. doi: 10.3390/medicina55090525. PMID: 31450833; PMCID: PMC6780141.

10.Qi W, Gevonden M, Shalev A. Prevention of Post-Traumatic Stress Disorder After Trauma: Current Evidence and Future Directions. Curr Psychiatry Rep. 2016 Feb;18(2):20. doi: 10.1007/s11920-015-0655-0. PMID: 26800995; PMCID: PMC4723637.

11. Van Ameringen M, Mancini C, Patterson B, Boyle MH. Posttraumatic stress disorder in Canada. CNS Neurosci Ther. 2008 Fall;14(3):171-81. doi: 10.1111/j.1755-5949.2008.00049.x. PMID: 18801110; PMCID: PMC6494052.

12.Xi Y, Chen R, Yan F, Ma X, Rakofsky JJ, Tang L, Guo H, Wu X, Li X, Zhu H, Guo X, Yang Y, Li P, Cao X, Li H, Li Z, Wang P, Xu Q, Tang Y, Broome MR. Low post-traumatic stress disorder rate in Chinese in Beijing, China. Asian J Psychiatr. 2017 Dec;30:7983. doi: 10.1016/j.ajp.2017.07.003. Epub 2017 Jul 4. PMID: 28837943.

13.MP. Shannon, CJ. Lonigan, et al. "Post-Traumatic Stress Disorder in Children and Adolescents One Year after a Super-Cyclone in Orissa, India: Exploring Cross-Cultural Validity and Vulnerability Factors." BMC Psychiatry, BioMed Central, 1 Jan.
1998, bmcpsychiatry.biomedcentral.com/articles/10.1186/1471244X-7-8.

14."VA.gov: Veterans Affairs." Adult PTSD Self-Report Measures, 24 Sept. 2018, www.ptsd.va.gov/professional/assessment/adult-sr/ index.asp.

15.Hoppen, T. H., \& Morina, N. (2019). The prevalence of PTSD and major depression in the global population of adult war survivors: a meta-analytically informed estimate in absolute numbers. European journal of psychotraumatology, 10(1), 1578637. https:// doi.org/10.1080/20008198.2019.1578637

- Author

Sruthi Medepalli is a sophomore at Lynbrook High School in San Jose, CA. Her interests include psychiatry, psychology, neuroscience, and journalism. Sruthi aims to bridge the gap between scientific research and common media in order to stop the spread of misinformation and wants to pursue a major in the brain sciences with a minor in literature or journalism. 